\title{
Pierre Bourdieu et la religion. Synthèse critique d'une synthèse critique
}

Erwan Dianteill

\section{(2) OpenEdition \\ 12 Journals}

Édition électronique

URL : http://journals.openedition.org/assr/1590

DOI : 10.4000/assr. 1590

ISSN : $1777-5825$

Éditeur

Éditions de l'EHESS

\section{Édition imprimée}

Date de publication : 1 avril 2002

Pagination : 5-19

ISBN : 2-222-96718-X

ISSN : 0335-5985

\section{Référence électronique}

Erwan Dianteill, «Pierre Bourdieu et la religion. Synthèse critique d'une synthèse critique », Archives de sciences sociales des religions [En ligne], 118 | avril - juin 2002, mis en ligne le 14 novembre 2005,

consulté le 30 avril 2019. URL : http://journals.openedition.org/assr/1590 ; DOI : 10.4000/assr.1590 


\section{PIERRE BOURDIEU ET LA RELIGION SYNTHÈSE CRITIQUE D'UNE SYNTHÈSE CRITIQUE}

La religion a un statut d'objet paradoxal dans l'œuvre de Pierre Bourdieu. Les articles qui y sont directement consacrés sont fort peu nombreux et aucun de ses ouvrages importants ne porte sur ce sujet. Comparée à la sociologie de l'art, de la culture, de l'éducation, du pouvoir ou de la misère sociale, la sociologie des religions occupe donc une place marginale dans le corpus. D'un autre côté, certains des concepts les plus importants du "structuralisme génétique » sont issus des sciences sociales des religions. Hérité de Mauss et Durkheim, le concept de croyance, qui est un ressort de tout champ, en est l'exemple le plus manifeste. Même l'élaboration de ce dernier concept, au dire du sociologue, provient de la rencontre entre ses recherches de sociologie de l'art, commencées vers 1960, et « le commentaire du chapitre consacré à la sociologie religieuse dans Wirtschaft und Gesellschaft», de Weber. "J'ai construit, écrit-il, la notion de champ à la fois contre Weber et avec Weber, en réfléchissant sur l'analyse qu'il propose des rapports entre prêtre, prophète et sorcier » (1987a, p. 33, p. 63). Autre exemple : c'est dans la lecture du travail de Panofski sur l'architecture gothique et la pensée scolastique que s'est forgée la définition de l'habitus (ibid., p. 23). De ce point de vue, l'œuvre de P. Bourdieu est presque une sociologie des religions "généralisée ", la religion présentant de façon paradigmatique des propriétés communes à toutes les sphères d'activité symbolique.

L'objet du texte ici présent, dont le titre est une forme d'hommage à une pensée qui s'est toujours voulue réflexive, est de présenter l'apport de P. Bourdieu aux sciences sociales des religions en parcourant à la fois son œuvre d'anthropologue et de sociologue. Peut-on parler de «religion » dans des sociétés où les religions institutionnelles n'existent pas ou dans les sociétés où elles sont singulièrement affaiblies? La notion de champ religieux reste-t-elle pertinente dans des sociétés non segmentées ou dans des sociétés de plus en plus sécularisées ? En d'autres termes, le " religieux » se limite-t-il au champ religieux ?

La lecture des œuvres de $\mathrm{P}$. Bourdieu suggère des réponses négatives à ces questions. La validité sociologique de la notion de champ religieux est limitée, en amont, par l'absence de monopolisation de la production symbolique dans certaines sociétés agraires, en aval, par l'incertitude croissante portant sur les limites du 
champ, due notamment à l'apparition de nouvelles professions spécialisées dans le « travail symbolique » et au "découplage » entre croyance et allégeance institutionnelle. Dans les travaux ethnologiques de P. Bourdieu, il apparaît que le symbolique informe l'ensemble de la vie sociale, sans que l'existence d'une institution autonome puisse permettre de parler de " champ religieux » (1980). Comment se constitue donc l'espace institutionnel religieux, à partir d'un espace social peu différencié (1971a) ? Le champ religieux semble en fait correspondre précisément aux religions historiques occidentales, notamment au judaïsme et au catholicisme analysés dans la dialectique entre relations internes et relations externes (Bourdieu et Saint-Martin, 1982), et l'on peut observer une certaine «dissolution du religieux » dans les sociétés occidentales aujourd'hui (1987c). On reviendra en conclusion sur le paradoxe qui a été relevé plus haut et dont l'élucidation permet de voir les limites de la sociologie des religions de P. Bourdieu : pourquoi, alors que l'étude des faits religieux est à l'origine de ses principaux concepts, occupe-t-elle une place quantitativement aussi marginale dans cette œuvre?

\section{La genèse sociale du champ religieux}

Il existe, selon P. Bourdieu, trois grandes théories sociologiques de la religion, symbolisées par trois noms: Marx, Weber, Durkheim. Ces théories semblent s'exclure mutuellement. Il s'agit donc de «se situer au lieu géométrique des différentes perspectives, c'est-à-dire au point d'où se laissent apercevoir à la fois ce qui peut et ce qui ne peut pas être aperçu à partir de chacun des points de vue » (1971a, p. 295). Que faut-il donc retenir de la pensée des trois perspectives ? L'apport de Durkheim est exprimé explicitement par P. Bourdieu, ceux de Marx et de Weber semblent moins clairement distingués.

P. Bourdieu retient des Formes élémentaires de la vie religieuse de Durkheim que la sociologie de la religion doit être considérée comme une dimension de la sociologie de la connaissance. La religion est un instrument de communication et un instrument de connaissance ; elle permet un accord sur le sens des signes et le sens du monde. Elle a une fonction d'intégration logique et sociale des « représentations collectives » et en particulier des "formes de classification » religieuses (ibid., p. 297).

L'apport wébérien à la sociologie du champ religieux est décisif car il permet d'échapper à l'alternative stérile entre le subjectivisme religieux et la réduction marxiste sans médiation (1971b, p. 1). De Weber vient ainsi l'idée que la sociologie religieuse est une dimension de la sociologie du pouvoir; il faut rattacher le discours mythique aux intérêts religieux de ceux qui le produisent, qui le diffusent et qui le reçoivent. Il existe une genèse historique des corps de spécialistes religieux, les clercs, ce qui constitue le fondement de l'autonomie relative du champ religieux. Ces professionnels de la religion ont des stratégies pour s'approprier le monopole de la contrainte hiérocratique, des biens de salut. Le champ religieux se présente donc comme le système complet des relations objectives de concurrence ou de transaction entre les positions des agents religieux (ibid., p. 6). 
Enfin il semble que P. Bourdieu retient de Marx la notion d'idéologie comme « transfiguration des rapports sociaux en rapports surnaturels, donc inscrits dans la nature des choses et par là justifiés »(1971a, p. 300). La religion assume, dans cette perspective une fonction politique de conservation de l'ordre social. Notons qu'il est difficile de différencier les apports de Marx et de Weber dans la mesure où l'un et l'autre situent la sociologie de la religion aux confins de la sociologie économique et de la sociologie politique.

Dans Le Sens pratique, les développements sur la magie sont surtout proches de la problématique durkheimienne et plus précisément maussienne. Dans «Genèse et structure du champ religieux », Weber et Marx dominent.

\section{Le symbolique omniprésent dans les sociétés agraires : Le Sens pratique}

Dans Le Sens pratique, œuvre de synthèse des travaux anthropologiques de P. Bourdieu, il n'est guère question de «religion », mais souvent de rituels, de magie, d'institutions magiques et de force illocutionnaire, cas particulier du pouvoir symbolique (le mot "religion » est d'ailleurs absent de l'index thématique ainsi que le mot "symbole" [1980, pp. 469-475]). La société kabyle, organisée autour des travaux agricoles et d'un nombre limité d'activités artisanales (comme le tissage, activité spécifiquement féminine), ne connaît pas de groupe de clercs autonome, mais elle est intégralement structurée par le "démon de l'analogie », système de schèmes constitués d'oppositions binaires dont la partition originaire « oppose le masculin et le féminin, le sec et l'humide, le chaud et le froid » (ibid., p. 367). Proche du structuralisme de Lévi-Strauss dans l'analyse des principes logiques organisant «la pensée sauvage » (Lévi-Strauss, 1962), P. Bourdieu s'en distingue par une attention accrue pour les dispositions incorporées qui génèrent des pratiques symboliques imparfaitement systématiques.

Selon lui, on peut comprendre complètement " toutes les pratiques et tous les symboles rituels à partir de deux schèmes opératoires qui, en tant que processus naturels culturellement constitués dans et par la pratique rituelle, sont inséparablement logiques et biologiques comme les processus naturels qu'ils visent à reproduire (au double sens) lorsqu'ils sont pensés dans la logique magique : d'une part la réunion des contraires séparés, dont le mariage, le labour ou la trempe du fer sont des actualisations exemplaires et qui engendre la vie, comme réunion réalisée des contraires, et, d'autre part, la séparation des contraires réunifiés, destruction et mise à mort, avec par exemple le sacrifice du bœuf et la moisson comme meurtres déniés » (1980, pp. 366-367). Mais si la logique rituelle demande la réunion ou la séparation des contraires, elle impose aussi de rendre socialement acceptable la transgression qu'elle signifie objectivement.

La magie fonctionne ainsi comme dénégation collective d'actes de transgression nécessaire (jonction du disjoint ou scission de l'uni). Sans ceux-ci les contraires séparés resteraient stériles. La transgression permet la reproduction de l'ordre vital, la reproduction du groupe, mais elle est excessivement dangereuse et demande donc une mise en forme collective, publique et pratique déniant le sens objectif du rite. Comment ces exigences contraires peuvent-elles être conciliées rituellement? Le sens pratique à l'œuvre dans la magie légitime des rites de licitation est en fait un double sens : affirmation de l'unité dans la séparation des con- 
traires; affirmation de la séparation dans leur unification. La conciliation de principes contradictoires ne peut être réalisée que dans l'autorisation circulairement accordée au groupe par le groupe au moment du rituel (1980, p. 385) :

Toute la vérité de la magie et de la croyance collective est enfermée dans ce jeu de la double vérité objective, dans ce double jeu avec la vérité, par lequel le groupe, responsable de toute objectivité, se ment en quelque sorte à lui-même, en produisant une vérité qui n'a de sens et de fonction que de nier une vérité connue et reconnue de tous, mensonge qui ne tromperait personne si tout le monde n'était résolu à se tromper. Dans le cas de la moisson, la vérité sociale qu'il s'agit de dénier collectivement est sans ambiguïté : la moisson (thamegra) est un meurtre (thamgert', désigne la gorge, la mort violente, la vengeance ; et amgar, la faucille) au terme duquel la terre, fécondée par les labours, est dépouillée des produits qu'elle a portés à leur maturité.

Les rites licitatoires qui impliquent une solution de continuité (déniée) dans la vie sociale kabyle ont pour pendants des rites propitiatoires. Leur logique est celle de « la gestion » de l'antagonisme qui menace l'ordre social et naturel. Ils permettent la transition entre les principes opposés, en particulier le passage sans heurt d'une période de l'année à une autre. C'est la féminisation du masculin à l'automne, la masculinisation du féminin au printemps, l'été et l'hiver étant symboliquement purement masculin et féminin.

Dans Le Sens pratique, la valeur heuristique de la distinction entre magie et religion est déniée, car cette opposition est conçue comme un enjeu de lutte symbolique étranger à la société kabyle. L'usage du terme " magie » vise, dans les sociétés segmentées, à disqualifier les pratiques symboliques des dominés, les dominants se réservant le terme de «religion ». Ces catégories sont inséparables de la constitution d'un champ religieux, avec ses enjeux concurrentiels entre prêtres, sorciers, et prophètes en Europe et au Proche-Orient. Elles ne sont pas pertinentes dans les sociétés peu segmentées (1980, p. 395) :

L'institution de périodes ou de moments licites (lah'lal), la désignation de mandatairesécrans (famille chargée d'ouvrir les labours, mariage inaugural de cousins parallèles) et l'organisation de grandes cérémonies collectives, dans lesquelles le groupe s'autorise de sa propre autorité, sont trois aspects de la même opération, qui est constitutive de tout rituel légitime (on embrouille tout en identifiant la distinction entre magie légitime et magie illégitime à la distinction, qui est un enjeu de luttes sociales, entre religion et magie). C'est sur l'autorité circulairement autorisée que le groupe s'accorde à lui-même, dans son entier ou en la personne de l'un des siens, mandataire autorisé, que repose la force illocutionnaire qui est à l'œuvre dans tous les rituels sociaux.

On retrouve dans les analyses précédentes des accents très clairement maussiens. La magie a un fondement social, la croyance, et une efficacité sociale qui lui est associée. «En définitive, écrit M. Mauss, c'est toujours la société qui se paie elle-même de la fausse monnaie de son rêve. La synthèse de la cause et de l'effet ne se produit que dans l'opinion publique. (...) Nous devons considérer la magie comme un système d'inductions a priori, opérées sous la pression du besoin par des groupes d'individus » (1985 [1902], p. 119). D'autre part, elle est pour Mauss et Hubert «à la fois un opus operatum au point de vue magique et un opus inoperans au point de vue technique » (1985 [1902]), p. 135), idée reprise et généralisée par P. Bourdieu (1980, p. 395) :

Le caractère proprement magique de cette force de part en part sociale échappe aussi longtemps qu'elle s'exerce seulement sur le monde social, séparant et unissant des individus ou des groupes par des frontières ou des liens (mariage) non moins magiques 
que ceux qu'instituent le couteau ou le nœud de la magie, transmuant la valeur sociale des choses (comme la griffe du couturier) ou des personnes (comme le titre scolaire). Il apparaît au contraire dans toute son évidence lorsque, par une sorte d'innocence, de confiance, de remise de soi imposée par la détresse et le désarroi extrêmes, les groupes tentent d'exercer le pouvoir qu'ils s'octroient eux-mêmes, par un de ces cercles qui sont au principe de la magie très réellement efficace du collectif, au delà de ses limites de validité, c'est-à-dire sur ce qui ne dépend pas d'eux, sur le monde naturel dont ils dépendent (...).

Au total, l'accent est porté dans les travaux anthropologiques de P. Bourdieu sur les conditions sociales d'efficacité du rite, en dehors d'un champ religieux non constitué dans la société kabyle. Il n’y a pas d'intermédiaires institutionnalisés (semble-t-il) entre le groupe et lui-même : il existe bien des familles chargées de couper la première gerbe au moment des moissons, par exemple, mais cette fonction n'est pas constante. Tout autre est le rôle social des institutions religieuses.

\section{Constitution des institutions religieuses : «Genèse et structure du champ religieux »}

Les «transformations technologiques, économiques et sociales»associées au développement urbain, comme « les progrès de la division du travail et l'apparition de la « séparation du travail intellectuel et du travail matériel» (1971a, pp. 300-301) sont à l'origine de deux processus intimement liés, que l'on pourrait qualifier de subjectif et d'objectif, à savoir la constitution d'un champ religieux d'une part, et d'autre part le processus de rationalisation des croyances et des rites. À la suite de Max Weber, P. Bourdieu rappelle les obstacles opposés à l'autonomisation des comportements religieux par la condition paysanne, alors que l'urbanisation favorise, par l'indépendance relative vis-à-vis des aléas naturels qu'elle procure, la « rationalisation » et la «moralisation » des besoins religieux (Weber, 1971, alinéa 7 : «Ordres, classes et religion »). De plus, l'urbanisation favorise le développement d'un corps de professionnels des biens de salut. Le clergé citadin contribue à l'intériorisation de la foi, à l'introduction de critères éthiques, du «bien » et du « mal », et de la notion de " péché », en tout cas dans le contexte judéo-chrétien. Il faut d'autre part attribuer à la conjonction des intérêts de la prêtrise et de ceux de certaines catégories de laïcs citadins, la domination du monothéisme en Palestine, et à Jérusalem en particulier.

Les deux processus précédents, à savoir d'une part la constitution d'un champ religieux relativement autonome, caractérisé par la production, la reproduction et la diffusion des biens et services religieux, et par une complexification institutionnelle croissante, et d'autre part un processus de " moralisation » des pratiques et des représentations religieuses, ont plusieurs corrélats. On passe ainsi du mythe à l'idéologie religieuse (monopolisation de la contrainte hiérocratique par un corps de professionnels), du tabou au péché (transfert de la notion de souillure de l'ordre magique à l'ordre moral), du Dieu punisseur au Dieu juste et bon (attribution de qualités de plus en plus « sociales » à la divinité) (1971a, p. 303).

Enfin la constitution d'un champ religieux s'accompagne d'une dépossession du capital religieux des laïcs au profit du corps de spécialistes religieux qui produisent et reproduisent un corpus délibérément organisé de savoirs secrets. On peut ainsi écrire que les différentes formations sociales se situent entre deux pôles: 
celui de l'autoconsommation religieuse et celui de la monopolisation complète de la production religieuse par des spécialistes. Ces deux positions extrêmes se caractérisent par l'opposition entre une maîtrise pratique d'un ensemble de schèmes de pensée et d'actions objectivement systématiques, acquis par simple familiarisation et une maîtrise savante, action pédagogique expresse et institutionnalisée. Elles sont aussi structurées par des systèmes mythico-rituels d'un côté, et des idéologies religieuses, réinterprétations lettrées des premiers en fonction d'intérêts internes ou externes liés à la constitution des États et aux antagonismes de classes (1971a, pp. 304-306).

Notons que même dans le cas des sociétés peu différenciées, P. Bourdieu propose d'importer les méthodes de la sociologie en ethnologie : celle-ci doit dépasser le culturalisme et concevoir la religion comme un fait social lié à d'autres faits sociaux notamment à la division du travail, elle doit savoir rapporter le discours mythique ou religieux à ses conditions sociales de production en s'attardant en particulier sur la formation et les caractéristiques des agents privilégiés de l'activité magico-religieuse. Ce qui ne signifie pas qu'il faille évacuer du champ de recherches les représentations collectives religieuses; il s'agit simplement de concevoir leur autonomie relative dans la structure sociale générale.

Enfin, les oppositions entre sacré et profane, entre religion et magie (que celle-ci se présente comme profanation objective, caractérisant la religion dominée, qui est le plus souvent issue d'un état ancien du champ religieux ; ou comme profanation intentionnelle, c'est-à-dire comme religion inversée) proviennent de l'opposition entre détenteurs du monopole du sacré et laïcs. En d'autres termes, on peut déduire des progrès de la division du travail religieux et de l'histoire du champ religieux les distinctions entre les catégories fondamentales de la pensée et de la pratique religieuse.

Cette genèse nous amène à considérer la structure et les enjeux essentiellement politiques du champ religieux, qu'il s'agisse des luttes internes ou de la relation entretenue avec l'espace social, relation qui constitue ce champ aujourd'hui comme structure structurante et structurée en voie de déstructuration.

\section{Structure et déstructuration du champ religieux}

L'autonomisation du champ religieux n'implique pas une indépendance absolue vis-à-vis des autorités temporelles, en particulier politiques. Dans la sociologie de P. Bourdieu, très influencée sur ce point par le marxisme, peut-être même dans sa version althussérienne, pratiques et représentations religieuses contribuent d'abord à informer une "vision du monde » essentiellement conservatrice, elles absolutisent le relatif et légitiment l'arbitraire de la domination. D'autre part, à partir de l'origine sociale du personnel religieux et de sa trajectoire dans l'institution, dont un exemple est donné par la structure et l'histoire contemporaine du « corps » des évêques dans l'Église catholique française (Bourdieu et Saint-Martin, 1982), le sociologue tente d'expliquer à la fois l'homogénéité de l'épiscopat et la division du 
travail religieux en son sein, cette dernière lui permettant de répondre à la demande religieuse. Enfin, quelques textes portent sur la dissolution du champ religieux, dont les frontières semblent de plus en plus floues dans les sociétés les plus modernes.

\section{Intérêts religieux et fonction sociale du religieux (relations internes, relations externes)}

Il existe un intérêt proprement religieux, que l'on peut définir comme «l'intérêt qu'un groupe ou une classe trouve dans un type déterminé de pratique ou de croyance religieuse et, en particulier, dans la production, la reproduction, la diffusion et la consommation d'un type déterminé de biens de salut (parmi lesquels le message religieux lui-même). Ce groupe social recherche la légitimation des propriétés symboliques et matérielles qui le caractérisent dans la structure sociale. P. Bourdieu refuse toute explication psychologique ou phénoménologique du fait religieux. Celui-ci doit être conçu « de manière proprement sociologique, i.e. comme l'expression légitimatrice d'une position sociale»(1971a, pp. 311-312). L'inspiration marxiste apparaît ici encore clairement. Elle s'est doublée dans les années 1990 de l'affirmation que la religion joue aussi un rôle dans la légitimation de la domination des hommes sur les femmes. L’Église, écrit-il sans nuance, est « habitée par l'antiféminisme profond du clergé » et elle " inculque (ou inculquait) explicitement une morale familialiste entièrement dominée par les valeurs patriarcales » (1998, pp. 92-93). Ajoutons que dans certains cas, la religion (ou plus exactement la magie) peut aussi permettre de trouver une solution (illusoire) à la misère sociale. «L'espérance magique est la visée de l'avenir propre à ceux qui n'ont pas d'avenir (...). Le millénarisme révolutionnaire et l'utopie magique sont la seule visée du futur qui s'offre à une classe dépourvue de futur objectif» (1977a, pp. 90-91). De nature différente pour les dominants et les dominés, l'intérêt religieux est le principal opérateur d'homologie entre le champ religieux et la structure générale des rapports sociaux.

Relativement autonome par rapport à la structure sociale, le champ religieux est en effet structuré par des enjeux internes et par des enjeux externes. Les positions de pouvoirs dans le champ résultent de la confrontation de la "demande religieuse (i.e. les intérêts religieux des différents groupes ou classes de laïcs) et de l'offre religieuse (i.e. les services religieux plutôt orthodoxes ou hérétiques) »(1971a, p. 319). La position de pouvoir qu'occupe une instance religieuse dans le champ dépend du pouvoir du groupe social dont elle s'est concilié l'appui. Cet appui, dans une relation dialectique, dépend à son tour de la position du groupe des producteurs dans le champ. Cette relation explique l'homologie de structure observée entre la structure sociale et le champ religieux : les dominants du champ religieux, par exemple, fondent leur domination sur celle des classes dominantes dans la structure sociale, tandis que les prophètes s'appuient sur les groupes dominés pour modifier l'état du rapport de forces dans le champ. Aussi, l'action du prophète est-elle à la fois un ferment de restructuration du champ religieux et de la structure sociale. Il apparaît donc que le «fonctionnement » du champ religieux est le produit d'une concurrence interne entre différentes instances (dont les principa- 
les sont l'Église, les prophètes, les sectes et les sorciers) en relation avec les lignes de force de la structure sociale.

Examinons un cas particulier étudié par P. Bourdieu : le groupe des évêques français à la fin des années 1970 .

\section{Origine sociale des évêques et effets sur l'organisation de l'Église catholique}

Le travail conjoint de P. Bourdieu et de Monique de Saint-Martin (1982) explore un nombre limité de dimensions ouvertes par les articles de 1971. Il s'agit principalement de saisir le processus menant l'ensemble des évêques à dénier toute différenciation interne correspondant à leur origine sociale. Dépassant l'image d'homogénéité produite par le corps de professionnels, on peut associer la classe sociale d'origine à la position occupée dans l'institution ecclésiastique, à condition de prendre en compte les déformations structurales, "les mêmes dispositions pouvant conduire à des positions et à des prises de positions différentes, voire opposées, dans des états du champ différents, ce qui a pour effet d'affaiblir, voire d'annuler la relation statistique avec la classe d'origine ». Il n'y a pas, en d'autres termes, de rapport mécanique entre l'origine sociale et la position au sein de l'Église.

Sous ces conditions, l'analyse fait apparaître ces deux groupes dans le corps épiscopal : «d'un côté les « oblats » qui, voués et dévoués à l'Église dès leur prime enfance, investissent totalement dans une institution à laquelle ils doivent tout; qui sont disposés à tout donner à une institution qui leur a tout donné, sans laquelle et hors de laquelle ils ne seraient rien. De l'autre, les évêques qui, ordonnés plus tard, possédaient, avant leur entrée dans l'Église, outre un capital social hérité, un capital scolaire important, et qui entretiennent de ce fait un rapport plus distant, plus détaché, et moins tourné vers les choses temporelles, à l'égard de l'institution, de ses hiérarchies, de ses enjeux »(1982, pp. 4-5). Les prises de positions adoptées par ces deux groupes ne sont intelligibles que si l'on prend en compte l'évolution historique. Le même habitus « aristocratique » caractérisant les « héritiers », qui aurait défini autrefois un rôle d'évêque hiératique et solennel, peut conduire aujourd'hui à éviter des postes trop communs, à accepter des évêchés « missionnaires », ou à rechercher le statut de théologien. L'opposition entre les deux catégories est formelle et non substantielle : « les antagonistes (...) peuvent échanger, en toute bonne foi, leurs positions : lorsque l'un d'eux, souvent le dominant, qui a le privilège des audaces, vient à changer, l'autre ne peut maintenir l'opposition qu'en changeant aussi » (ibid., p. 16).

Comment se situe le corps des évêques «dans le champ du pouvoir religieux et, plus largement dans le champ du pouvoir symbolique » (ibid., p. 22) ? Les évêques sont en quelque sorte "pris entre deux feux »: ils s'opposent d'une part au pouvoir politique central des organismes ecclésiastiques, peu enclins à prêter attention aux réalités locales, d'autre part aux théologiens et religieux, orientés vers « le pouvoir symbolique central (...) moins soucieux des choses temporelles qui préoccupent les "responsables" » (ibid., p. 28). Assumant cette position moyenne dans l'Eglise catholique, les évêques peuvent remplir pleinement le travail d'unification qui leur incombe. Leur cohésion est renforcée par des dispositions communes (ce sont tous des hommes, souvent issus de familles nombreuses et nés dans de petits 
villages) et par une formation homogénéisante. Aussi, l'épiscopat est-il un champ de concurrence, mais à faible tension (ibid., p. 31).

L'ajustement entre l'offre et la demande religieuse n'est pas le produit d'une transaction, comme pouvait le penser Max Weber, mais l'effet d'une homologie involontaire de structure, "chaque clerc produisant en fonction de sa trajectoire et de sa position un produit plus ou moins ajusté à la demande d'une catégorie particulière de laïcs » (ibid., p. 34). L’Église, en tant que champ, se caractérise par l'unité et la diversité. Cette apparente contradiction est en fait au principe de sa grande capacité d'adaptation: elle permet à l'Église de «traiter de façon aussi homogène que possible une clientèle distribuée (de manière différente selon les époques) entre les classes sociales, les classes d'âge et les classes sexuelles ou de traiter de manière aussi différenciée que possible des clients qui, quoique très différents sous différents rapports, ont en commun d'être catholiques « (ibid., p. 35). Cette capacité à traiter des demandes variées sous les apparences de l'unité est renforcée par le caractère intrinsèquement polysémique du discours religieux, qui masque sous un discours unique une pluralité de significations en rapport avec une série de positions sociales, renforçant ainsi la confusion subjective de limites sociales objectives. Le discours religieux tend à nier les conflits sociaux, ou faute de mieux, à les euphémiser. P. Bourdieu avance même que les procédés discursifs du double sens et de l'euphémisme sont «profondément caractéristiques du discours religieux dans son universalité » (ibid., p. 46).

Cette transfiguration des rapports de forces, en particulier économiques, par les agents religieux dominants est illustrée par la gestion par l'épiscopat des revendications professionnelles de laïcs travaillant pour l'Église à la limite du bénévolat et du salariat. L'économie de l'Église est déniée par la hiérarchie en tant qu'économie régie par des lois économiques, c'est-à-dire fondée sur le prix, le salaire, l'offre et la demande. «Le rire des évêques », lorsqu'ils sont exposés à un discours qui traite l'Église comme une société commerciale, est significatif de la «vérité de l'entreprise religieuse (qui) est d'avoir deux vérités : la vérité économique et la vérité religieuse, qui la dénie»(1994a, p. 204). L'hilarité épiscopale est un effet du dévoilement de ce qui leur apparaît comme une parfaite incongruité (mais qui n'est pas sans fondement puisque l'on peut considérer, du point de vue du marché du travail, que la chaisière est une femme de ménage sans salaire).

Au total, P. Bourdieu conçoit donc l'Église comme un ensemble de mécanismes et de processus de légitimation des positions sociales, se présentant sous une forme objectivée, qu'elle soit matérielle (bâtiments, vêtements, instruments liturgiques, etc.) ou à l'état de technologie sociale (droit canon, liturgie, théologie, etc.). A l'état incorporé, elle est consubstantielle aux habitus catholiques, générés par la famille chrétienne et consacrés par des rites d'institution, qui agrègent tout en séparant. « Et en définitive, elle n'existe vraiment en tant qu'Église vivante, c'est-à-dire agissante et capable d'assurer sa propre reproduction, que dans la relation entre ses deux modes d'incarnation (...) » (Bourdieu et Saint-Martin, 1982, p. 51).

\section{Vers une dissolution du champ religieux ?}

Sans parler d'évolutionnisme au sens classique du terme, la sociologie des religions de P. Bourdieu se caractérise aussi par une grande attention accordée aux 
processus historiques de constitution des institutions religieuses. Ceux-ci ne se réalisent pas indépendamment des changements généraux affectant la structure sociale, notamment des progrès de la division du travail social et de l'urbanisation. P. Bourdieu n'utilise pas le concept de "champ religieux » pour les sociétés agraires comme la société kabyle, du fait, selon lui, du manque d'institutions et de professionnels spécialisés. Peut-on alors parler de «religion» s'il n'existe pas de « champ religieux »? On ne peut parler de l'activité symbolique dans les mêmes termes pour des sociétés agraires peu différenciées, où le "démon de l'analogie » informe et unifie toutes les dimensions de la structure sociale, et pour des sociétés très segmentées, où la production symbolique se concentre dans des espaces institutionnels spécifiques et relativement autonomes dont le champ religieux constitue l'un des principaux.

Cependant, les sociétés modernes qui se caractérisent par un haut degré de division du travail social, sont en même temps celles où les religions historiques, et en particulier le catholicisme, semblent en déclin. On y assiste à une "dissolution du religieux » (1987c, pp. 117-123). «(...) On assiste à une redéfinition des limites du champ religieux, la dissolution du religieux dans un champ plus large s'accompagnant d'une perte du monopole de la cure des âmes au sens ancien, du moins au niveau de la clientèle bourgeoise » (Bourdieu, 1985, p. 257). Les limites du champ religieux ne sont plus clairement définies : certaines professions profanes, psychologues, analystes ou conseillers conjugaux, se substituent aux clercs dans leur fonction thérapeutique. Ainsi, « le champ religieux s'est trouvé dissout dans un champ de manipulation symbolique plus large «(Bourdieu, 1985, p. 259). Dans un dialogue avec Jacques Maître, auteur de plusieurs ouvrages de psychopathologie sociale appliquée à des individus fortement impliqués dans le catholicisme, P. Bourdieu déclare qu' " il se pourrait que la psychanalyse ait aujourd'hui, dans la conscience commune, une fonction assez analogue à ce qu'était la religion pour » les personnages étudiés par J. Maître (Bourdieu et Maître, 1994, p. XVI).

D'un autre côté, le refus de l'obéissance aveugle aux prescriptions des clercs provient pour une part de l'élévation du niveau d'instruction, qui entraîne moins le rejet d'une "posture » religieuse que celui de la délégation spirituelle. Ce déni (relatif) de la légitimité accordée à l'institution catholique contribue ainsi au développement de sectes autonomes, au « rassemblement de petits prophètes charismatiques », et plus généralement à la disjonction entre l'orthodoxie cultuelle et les pratiques et croyances effectives. Ainsi, la légitimité de la parole religieuse d'institution est en concurrence avec de nouvelles formes de légitimité et de nouvelles professions, qui s'appuient souvent sur un discours pseudo-scientifique, comme les astrologues, numérologues ou graphologues par exemple. Le clerc religieux, anciennement dominant, devient dominé, « au profit de clercs qui s'autorisent de la science pour imposer des valeurs et des vérités ni plus ni moins scientifiques que celles des autorités religieuses du passé »(1987c, p. 123).

L'émergence d'un sentiment esthétique face aux représentations visant à l'origine à susciter la foi est aussi un indice de la régression de la «croyance religieuse » par rapport à la «croyance esthétique ». En un même lieu (l'Église Santa Maria Novella observée par le sociologue en 1982), se juxtaposent des pratiques qui tiennent à la fois de la muséographie et de la dévotion, et qui témoignent de l'hétérogénéité des visées du public dans l'«admiration »d'une Vierge au Rosaire ou d'une Présentation au Temple. L'usage dévotionnel des statues et des tableaux 
n'a pas pourtant complètement disparu, mais il se concentre sur les œuvres dont les caractéristiques sont les moins formelles, celles qui «remplissent une fonction expressive de représentation de leurs référents » (1994c, p. 73).

Remarquons enfin que la «dissolution du religieux» ne signifie pas pour le sociologue critique une régression à un état indifférencié de l'activité symbolique. Les «nouveaux agents symboliques », qui se situent en dehors de l'institution religieuse, coexistent avec une institution catholique qui tend à devenir « une Église sans fidèles » (1994b, p. 216), s'y opposent souvent, mais contribuent aussi à faire évoluer les positions de celle-ci, comme en témoigne par exemple l'influence de la psychanalyse sur la pensée religieuse moderne. L'activité symbolique aux marges du champ religieux engendre une certaine confusion quant à ses limites, mais elle ne signifie pas pour autant sa disparition ${ }^{1}$.

\section{Conclusion : dignité et indignité de la religion comme objet de science sociale}

Le pouvoir symbolique, qui ne se limite certes pas au domaine religieux, s'y exerce peut-être plus clairement que dans d'autres champs d'activité sociale, et c'est probablement pourquoi une bonne partie de l'architecture conceptuelle de la sociologie de P. Bourdieu s'est construite dans l'étude de la religion. Le pouvoir symbolique est en effet ce qui permet de constituer le donné par l'énonciation, « de faire voir et de faire croire, de confirmer ou de transformer la vision du monde et par là, l'action sur le monde, donc le monde ». C'est « un pouvoir quasi-magique qui permet d'obtenir l'équivalent de ce qui est obtenu par la force (physique ou économique). Ce pouvoir ne s'exerce que s'il est reconnu, c'est-à-dire méconnu comme arbitraire » (Bourdieu, 1977b, p. 410). La religion apparaît donc, dans cette perspective, comme une activité essentiellement symbolique, comme une forme symbolique, c'est-à-dire comme un ensemble de pratiques et de représentations (en l'occurrence de rites et de croyances) dont l'efficacité n'est pas d'ordre matériel (comme la force physique par exemple). Le symbolique comprend le langage mais ne s'y réduit pas; il désigne aussi la fonction connotative des signes, linguistiques ou non. Le pouvoir du symbole repose sur ce qu'il ne dit pas explicitement, sur ce qu'il suppose sans l'expliciter ouvertement. Il induit un rapport de sens socialement fondé, ce qui signifie que le pouvoir symbolique ne se constitue pas en dehors des rapports de forces caractéristiques de la structure sociale dans sa totalité. Si le symbole a une efficacité propre, elle n'en est pas moins en rapport avec la genèse et la structure de l'espace social. Plus précisément, pour ce qui concerne le pouvoir symbolique du langage, son efficacité nécessite "la croyance dans la légitimité des mots et de celui qui les prononce, croyance qu'il n'appartient pas aux mots de pro-

${ }^{1}$ Il ne faut pas confondre cette activité symbolique de substitution religieuse avec le pouvoir symbolique " hors champ religieux », notamment celui de l'État en tant que banque centrale symbolique (1989) qui s'appuie sur des rites d'institution (1982). La magie sociale ne se limite donc pas au champ religieux, comme en témoigne par exemple la force purement symbolique des « marques » et des « griffes » dans la haute couture (1984). 
duire » (Bourdieu, 1977b, p. 411). En d'autres termes, le pouvoir symbolique ne se suffit pas à lui même, il trouve son fondement dans les rapports généraux de domination, dont il peut apparaître comme une "sublimation ».

Entre efficacité propre et dépendance idéologique, entre ordre sui generis et superstructure, le fait religieux n'est dans cette perspective ni purement idéal, ni simple reflet plus ou moins déformé de la structure sociale. Le secret de cette dépendance/indépendance du pouvoir symbolique religieux est à chercher dans cette instance intermédiaire, dont l'analyse constitue le centre de la sociologie religieuse de P. Bourdieu : la notion de champ religieux. La notion d'habitus complète la précédente en ce qu'elle associe à un champ spécifique un type d'intérêt spécifique, irréductible aux intérêts des autres champs, notamment à l'intérêt économique. " Pour qu'un champ marche, il faut qu'il y ait des enjeux et des gens prêts à jouer le jeu, dotés de l'habitus impliquant la connaissance et la reconnaissance des lois immanentes du jeu, des enjeux, etc. » (1984a, p. 114). Un intérêt spécifiquement religieux est donc associé au champ religieux, qui donne un exemple particulièrement achevé de cette «liberté surveillée» caractéristique des instances secondes dans les mécanismes de la domination (où l'économique, le politique et le médiatique semblent occuper les positions centrales, du moins dans les sociétés participant de la modernité avancée).

Pour P. Bourdieu, le champ religieux ne doit donc pas être conçu comme une réalité immuable : il existe une genèse structurale du champ religieux, en rapport avec les transformations de la structure sociale, et l'on assiste, selon lui, à une dissolution du religieux dans les sociétés en voie de sécularisation. Alors que toute institution religieuse tend à se présenter comme réalité anhistorique, identique à elle-même de tout temps et en tout lieu, il faut passer par l'examen historique des processus de constitution des croyances, des rites, des institutions.

On peut alors se demander pourquoi la sociologie des religions, qui a fourni à P. Bourdieu une grande partie de son architecture conceptuelle et dont il ignore moins que tout autre qu'elle constitue le cœur de la sociologie de Durkheim, de Weber et une partie non négligeable de celle de Marx, a une importance si limitée en termes quantitatifs dans son œuvre.

Cet écart provient d'une incertitude fondamentale quant aux conditions de possibilité de l'étude scientifique de la religion. Pour P. Bourdieu, la religion est en fait un objet sociologique presque impossible. Cette " gène » épistémologique n'a jamais été plus manifeste qu'à l'occasion du colloque annuel de l'Association Française de Sociologie des Religions, en décembre 1982, lorsque le sociologue questionna publiquement la validité scientifique de la sociologie des religions lorsqu'elle est pratiquée par « des producteurs qui participent à des degrés divers au champ religieux » (1987b, p. 156). Pour un sociologue des religions, avoir des convictions religieuses constitue un obstacle presque insurmontable au travail scientifique. Mais il existe un deuxième obstacle, tout aussi dangereux : l'absence complète d'affinité avec l'objet. Selon P. Bourdieu, tout sociologue de la religion est ainsi confronté à une contradiction quasi-insoluble: "quand on en est, déclare-t-il, on participe de la croyance inhérente à l'appartenance à un champ quel qu'il soit (religieux, universitaire, etc.) et quand on n'en est pas, on risque premièrement d'omettre $d$ 'inscrire la croyance dans le modèle $(. .$.$) , deuxièmement$ d'être privé d'une partie de l'information utile » (1987b, p. 156). Le premier écueil, c'est-à-dire le fait d'appartenir d'une façon ou d'une autre à l'institution religieuse, 
peut mener à adopter un point de vue religieux sur la religion, à pratiquer une sociologie religieuse plus qu'une sociologie des religions. Pour l'éviter (ce qui est "difficile », mais pas impossible selon P. Bourdieu), il faut pratiquer "une objectivation sans complaisance (...) de tous les liens, de toutes les formes de participation, d'appartenance objective ou subjective, même les plus ténues » (ibid., p. 160). Le deuxième écueil (ne pas en être) n'est pas moins dangereux scientifiquement. Celui qui en est victime tombe dans l'ornière positiviste en abordant la religion exclusivement d'un point de vue extérieur, "comme une chose ", sans apercevoir les ressorts subjectifs de l'activité religieuse, et en particulier l'adhésion inconditionnée à des vérités révélées.

Or, il y a une affinité certaine entre cette seconde posture et les sciences sociales «républicaines ». La sociologie française s'est largement constituée contre l'emprise intellectuelle de la religion, et singulièrement contre l'influence catholique dans l'université, au moment des luttes anticléricales du début du $\mathrm{XX}^{\mathrm{e}}$ siècle. D'une certaine façon, être sociologue demandait nécessairement de «ne pas en être ». P. Bourdieu n'a pas échappé à cette forme d'anticléricalisme. Dans la conclusion du colloque sur "les nouveaux clercs», on peut lire par exemple cette déclaration sans ambiguïté : "L'interrogation sur les 'nouveaux clercs' n'aurait peut-être pas manqué son but si elle pouvait ainsi conduire à poser les fondements d'un nouvel anticléricalisme » (1985, p. 261). Notons que cette phrase conclusive n'a pas été reprise dans la deuxième version du texte publiée dans Choses dites (1987). Ce « repentir » témoigne assurément d'un certain malaise, dont P. Bourdieu a lui-même tenté de rendre raison dans un entretien avec Jacques Maître (1994, p. XV) :

J'ai eu à découvrir dans ma propre tête toutes les mutilations que j'avais héritées d'une tradition laïque, renforcée par les présupposés implicites de ma science. Il y a des sujets qu'on n'aborde pas, ou seulement avec la plus grande prudence. Il y a des manières d'aborder certains sujets qui sont un peu dangereuses et, finalement, on accepte les mutilations que la science a dû accepter pour se constituer. Tout ce qui est de l'ordre des objets traditionnels de la religion et de la métaphysique, on se sent tenu - par une adhésion implicite qui est liée à l'entrée dans la profession - de le mettre entre parenthèses. Il y a une espèce de refoulement qui est tacitement exigé du professionnel.

N'« en étant pas » par méfiance méthodologique et par culture laïque, P. Bourdieu manquait assurément d' " intérêt » pour le fait religieux, ce qui le plaçait dans une position peu favorable pour l'étudier sociologiquement. Dans la perspective de la sociologie critique et réflexive, on l'a vu, l'incrédulité militante est aussi dommageable scientifiquement que la « faiblesse de croire », car elle entrave toute restitution du sens des «adhésions» religieuses. Première victime de cette aporie, P. Bourdieu s'est donc investi de façon très limitée dans la sociologie des religions, d'autant plus qu'il considérait le pouvoir religieux, largement assimilé à celui de l'Église catholique, comme un pouvoir déclinant dont la critique est sans enjeux, contrairement par exemple à la domination masculine ou au pouvoir télévisuel. En d'autres termes, P. Bourdieu ne manifestait aucune inclination pour l'activité religieuse (alors que l'on connaît son goût pour l'art et la littérature, et bien sûr pour la pédagogie), mais cette distance était doublée de l'idée que la religion n'a plus l'emprise sociale qu'elle avait encore du temps de Weber ou Durkheim, qui en avaient fait l'objet central de leur sociologie. P. Bourdieu n'avait donc pas non plus d'intérêt négatif pour la religion (alors qu'il s'en est pris dans la dernière partie de 
son œuvre aux médias ou à la «Noblesse d'État», dont le pouvoir est conçu comme bien réel). C'est pourquoi, confronté à des contradictions méthodologiques difficiles à résoudre, pénétré par une culture laïque qui assimile la religion au pouvoir de l'Église catholique, convaincu que celle-ci constitue une force sociale déclinante, P. Bourdieu n'a accordé qu'une place marginale à l'étude des faits religieux dans son œuvre.

Remarquons pour finir que la lecture en parallèle de ses travaux anthropologiques et sociologiques suggère qu'il existe une division entre les sociétés agraires sans institution religieuse ${ }^{2}$ et les sociétés différenciées où la religion serait d'abord entièrement monopolisée par l'Église avant de disparaître en même temps qu'elle. Or, si l'Église catholique n'a plus le pouvoir politique et économique qu'elle a eu, cela signifie-t-il pour autant que croyances et pratiques religieuses ont disparu dans les sociétés modernes? Rien n'est moins sûr. Une définition de la «religion» centrée sur le « champ religieux », c'est-à-dire sur les luttes entre spécialistes dans leurs rapports avec la structure sociale globale, permet en fait difficilement d'étudier des phénomènes religieux qui échappent largement au contrôle des clercs, fussent-ils de «nouveaux clercs ». Cette erreur de perspective aurait pu être évitée en accordant plus de place au fait religieux hors institution, y compris dans les sociétés différenciées. En portant, par exemple, son attention sur le bricolage des croyances ou sur la construction des lignées de fidèles dans la modernité, sur l'organisation en réseau des amateurs d'ésotérisme ou sur l'organisation non bureaucratique de certains pentecôtismes, on peut éviter d'assimiler l'étude des faits religieux au simple constat de la perte de pouvoir des institutions ecclésiastiques.

\section{Erwan DIANTEILL}

Centre d'Études Interdisciplinaires des Faits Religieux EHESS

\footnotetext{
${ }^{2}$ Si la sociologie des religions de P. Bourdieu se focalise sur le catholicisme, son anthropologie kabyle minore singulièrement le rôle de l'islam. Le structuralisme, qui informe largement ces premiers travaux, s'intéresse avant tout aux systèmes symboliques, et non aux institutions religieuses. Ajoutons que l'islam était considéré comme une force sociale déclinante au moment de la décolonisation de l'Afrique du Nord, dans les années 1950 et 1960. Comme le catholicisme, l'idéologie laïque percevait alors la religion musulmane comme un archaïsme condamné par la modernité à plus ou moins long terme.
} 


\section{BIBLIOGRAPHIE}

Bourdieu Pierre, " Genèse et structure du champ religieux », Revue Française de sociologie, 1971a, vol. XII, no 2 , pp. 295-334.

Bourdieu Pierre, "Une interprétation de la théorie de la religion selon Max Weber », Archives européennes de sociologie, 1971b, vol 12, pp. 3-21.

Bourdieu Pierre, Algérie 60, Paris, Éditions de Minuit, 1977a.

BourdiEu Pierre, «Sur le pouvoir symbolique », Annales ESC, 1977b, vol. XXXII, no 3 (mai-juin), pp. 405-411.

Bourdieu Pierre, Le Sens pratique, Paris, Éditions de Minuit, 1980.

Bourdieu Pierre, Ce que parler veut dire, Paris, Fayard, 1982.

BourdiEu Pierre, « Haute culture et haute couture », in Questions de sociologie, Paris, Édition de Minuit, 1984a, pp. 196-206.

Bourdieu Pierre, "Quelques propriétés des champs », in Questions de sociologie, Paris, Éditions de Minuit, 1984b, pp. 113-120.

Bourdieu Pierre, «Le champ religieux dans le champ de manipulation symbolique », in Collectif, Les nouveaux clercs - Prêtres, pasteurs et spécialistes des relations humaines et de la santé, Genève, Labor et Fides, 1985, pp. 255-261 (repris partiellement sous le titre « La dissolution du religieux » dans Choses dites, Paris, Éditions de Minuit, 1987, pp. 117-123).

Bourdieu Pierre, «Fieldwork in philosophy» in Choses dites, Paris, Éditions de Minuit, 1987a, pp. 13-46.

Bourdieu Pierre, "Sociologues de la croyance et croyances de sociologues » Archives de Sciences Sociales des Religions, 63/1, janvier-mars 1987b, pp. 155-161 (repris partiellement dans Choses dites, Paris, Éditions de Minuit, 1987, pp. 106-111).

Bourdieu Pierre, «La dissolution du religieux » in Choses dites, Paris, Éditions de Minuit, 1987c, pp. 117-123.

Bourdieu Pierre, La Noblesse d'État - Grandes Écoles et esprit de corps, Paris, Éditions de Minuit, 1989.

Bourdieu Pierre, "Le rire des évêques ", in Raisons Pratiques - Sur la théorie de l'action, Paris, Seuil, 1994a, pp. 202-213.

Bourdieu Pierre, "Propos sur l'économie de l'Église », in Raisons Pratiques - Sur la théorie de l'action, Paris, Seuil, 1994b, pp. 215-217.

Bourdieu Pierre, "Piété religieuse et dévotion artistique », Actes de la Recherche en Sciences Sociales, décembre 1994c, no 105, pp. 71-74.

Bourdieu Pierre, La domination masculine, Paris, Seuil, 1998.

Bourdieu Pierre, Saint Martin Monique de, « La Sainte Famille : l’épiscopat français dans le champ du pouvoir », Actes de la Recherche en Sciences Sociales, 1982, n 44-45, pp. 1-53.

Bourdieu Pierre, Maître Jacques, «Avant-propos dialogué », in Jacques MAîTre, L'autobiographie d'un paranoïaque - L'abbé Berry (1878-1947) et le roman de Billy, "Introübo", Paris, Anthropos, 1994.

Durkheim Émile, Les formes élémentaires de la vie religieuse, Paris, Presses Universitaires de France, 1985 (1912).

LÉvi-Strauss Claude, La Pensée sauvage, Paris, Presses Universitaires de France, 1962.

Mauss Marcel, Henri Hubert, « Esquisse d'une théorie générale de la magie » in Sociologie et anthropologie, Paris, Presses Universitaires de France, 1985 (1902), pp. 1-141.

Weber Max, «Les types de communalisation religieuse (sociologie de la religion)» in Économie et société, Paris, Plon, 1971, pp. 429-632. 
